\title{
Antimatter Requirements and Energy Costs for Near-term Propulsion
} Applications

\author{
G.R. Schmidt*, H.P. Gerrish** and J.J. Martin*** \\ NASA Marshall Space Flight Center, Huntsville, Alabama 35812 \\ and \\ G.A. Smith $\dagger$ and K.J. Meyer $\dagger^{\dagger}$ \\ Pennsylvania State University, University Park, Pennsylvania 16802
}

\begin{abstract}
The superior energy density of antimatter annihilation has often been pointed to as the ultimate source of energy for propulsion. However, the limited capacity and very low efficiency of present-day antiproton production methods suggest that antimatter may be too costly to consider for near-term propulsion applications. We address this issue by assessing the antimatter requirements for six different types of propulsion concepts, including two in which antiprotons are used to drive energy release from combined fission/fusion. These requirements are compared against the capacity of both the current antimatter production infrastructure and the improved capabilities that could exist within the early part of next century. Results show that although it may be impractical to consider systems that rely on antimatter as the sole source of propulsive energy, the requirements for propulsion based on antimatter-assisted fission/fusion do fall within projected near-term production capabilities. In fact, a new facility designed solely for antiproton production but based on existing technology could feasibly support interstellar precursor missions and omniplanetary spaceflight with antimatter costs ranging up to $\$ 6.4$ million per mission.
\end{abstract}

I

\section{Nomenclature}

\footnotetext{
- Deputy Manager, Propulsion Research Center. Member AIAA.

** Research Engineer, Propulsion Research Center. Member AIAA.

** Research Engineer, Propulsion Research Center.

$\uparrow \quad$ Director \& Professor, Laboratory for Elementary Particle Science. Member AIAA.

ti Graduate Student, Dept of Aerospace Engineering.
} 


\begin{tabular}{|c|c|c|}
\hline$c$ & $=$ & speed of light \\
\hline$E_{\text {fusion }}$ & $=$ & energy contribution from fusion \\
\hline$E_{\text {in }}$ & $=$ & energy into antiproton production process \\
\hline$E_{\text {out }}$ & $=$ & antimatter rest mass energy out of production process \\
\hline$E_{\bar{p} p}$ & $=$ & energy contribution from antimatter annihilation \\
\hline Isp & $=$ & specific impulse \\
\hline$K$ & $=$ & total energy cost \\
\hline$k_{\text {grid }}$ & $=$ & power utility cost ( $\$$ per unit energy) \\
\hline$M_{a}$ & $=$ & mass of antiprotons \\
\hline$M_{o}$ & $=$ & vēhicle dry mass (structure + payload) \\
\hline$M_{p}$ & $=$ & propellant mass \\
\hline$M_{p a y}$ & $=$ & payload mass \\
\hline$R$ & $=$ & spacecraft wet mass to dry mass ratio \\
\hline$V_{e}$ & $=$ & rocket exhaust velocity \\
\hline$\beta$ & $=$ & fusion energy to annihilation energy ratio \\
\hline$\gamma$ & $=$ & Lorentz-Fitzgerald factor, $\gamma=\frac{1}{\sqrt{1-\left(V_{e} / c\right)^{2}}}$ \\
\hline$\Delta V$ & $=$ & mission velocity requirement \\
\hline$\eta_{\text {conr }}$ & $=$ & energy conversion efficiency \\
\hline$\eta_{\text {grid }}$ & $=$ & wall-plug efficiency \\
\hline$\eta_{t o t}$ & $=$ & total production efficiency \\
\hline$\eta_{e}$ & $=$ & propulsion energy utilization efficiency \\
\hline$\lambda$ & $=$ & vehicle structure to propellant mass ratio \\
\hline
\end{tabular}

\section{Introduction}

The annihilation of subatomic particles with their antimatter counterparts has the highest energy per unit mass of any reaction known in physics. The energy released from protonantiproton annihilation $\left(1.8 \times 10^{14} \mathrm{~J} / \mathrm{g}\right.$ of antiprotons) is $10^{10}$ times greater than oxygen-hydrogen combustion and at least 100 times more energetic than fission or fusion. That is, one gram of antihydrogen (i.e., "mirror" hydrogen atom composed of an antiproton and positron) reacted 
with the same amount of normal hydrogen produces a total energy equivalent to that delivered by 23 Shuttle External Tanks (ET).

Ever since 1953 when Eugene Sanger first proposed use of electron-positron annihilation to produce thrust, 'there have been many attempts ${ }^{2-6}$ to identify ways of exploiting antimatter for propulsion. Practically all of these concepts involve applying the products from protonantiproton annihilation either to create thrust directly, or to energize a propellant through interparticle collisions or heating of an intermediate solid core. In addition, the scientific community, which until several decades ago exhibited only casual interest in the subject, is now devoting more attention and resources to uses of antimatter. The best examples of this are the accelerators at Fermi National Accelerator Laboratory (FNAL) and The European Laboratory for Particle Physics (CERN), which routinely produce antiprotons to extend the energy range of particle collision experiments.

Although the worldwide production capacity has been growing at a nearly geometric rate since the discovery of the antiproton in 1955, the current output rate of 1 to 10 nanograms (ng) per year is minuscule compared to that of other exotic materials. For this reason, some people have questioned the practicality of using antimatter for propulsion, at least within the next century or so. They feel that the energy costs would be exorbitantly high and would never allow antimatter to be competitive with other propulsion technologies.

Most of this skepticism stems from the misconception that all the concepts that utilize antimatter rely on the annihilation reaction as the sole source of propulsive energy. Although it is true that "conventional" antimatter systems, which derive all their energy from annihilation, offer the highest specific impulse ( Isp $\sim 10^{5}$ to $10^{7} \mathrm{sec}$ ) of any propellant-based propulsion concept, there are several antiproton-driven hybrid fission/fusion concepts that require far less antimatter, while still coming close to the performance of conventional antimatter rockets (Isp $\sim 10^{4}$ to $\left.10^{6} \mathrm{sec}\right) .^{7.9}$ In fact, the quantities required to test and demonstrate these concepts may be well within the range of existing production facilities at FNAL and CERN, once several promising upgrades are incorporated.

It appears that the prospects of exploiting antimatter for space propulsion are not so bleak after all and may indeed be quite favorable. We have confirmed this by conducting a study in which we calculated the antimatter quantities required to accomplish a broad range of missions, and compared these values against the production costs of the current infrastructure. Using these numbers as a reference, we examined the incorporation of upgrades and improvements that could 
further increase production capacity and ultimately lower energy costs. The results suggest an evolution of production infrastructure. starting with quantities to support development of antimatter-assisted fission/fusion propulsion technology, followed by actual use of these systems for omniplanetary spaceflight and interstellar precursor missions beyond the heliopause. ${ }^{10}$

\section{Fundamental Energy Cost Constraints}

The creation of antimatter is an inherently energy-intensive process. Not only must energy be converted into rest mass of antiparticles, but the application of this energy is ordinarily inefficient and potentially quite expensive. The energy cost can be generally expressed as:

$$
K=k_{\text {grid }} E_{\text {grid }} \text {, }
$$

where $k_{\text {grid }}$ is the unit cost of energy, and $E_{\text {grid }}$ is the energy consumed at the wall-plug (i.e., off the commercial power grid).

The wall-plug energy $E_{\text {grid }}$ is related to the antimatter rest mass collected $M_{a}$ and the overall efficiency of converting wall-plug power into antimatter $\eta_{t o t}$ by:

$$
E_{\text {grid }}=M_{a} c^{2} / \eta_{t o t}
$$

The efficiency $\eta_{t o t}$ may be separated into two parts, that is:

$$
\eta_{\text {tot }}=\eta_{\text {conv }} \eta_{\text {grid }}
$$

where $\eta_{\text {conv }}$ is defined solely by features of the production and collection process, and $\eta_{g r i d}$ is the electrical efficiency of the accelerator system. Conservation of baryon number requires that formation of an antiparticle is always accompanied by creation of its standard particle counterpart. Thus, the antiproton can at most be $1 / 2$ of the total rest mass produced in a perfectly efficient conversion process. This sets a theoretical limit on $\eta_{\text {conv }}$ of $1 / 2 .{ }^{11}$

The total energy cost is obtained by substituting Eqs. (2) and (3) into Eq. (1), namely: 


$$
K=\frac{k_{g r i d} M_{a d} c^{2}}{\eta_{c o m} \eta_{g r r i d}}
$$

Equation (4) clearly shows that $\eta_{\text {com }}$ and $\eta_{\text {grid }}$ are major factors in dictating energy costs. Unfortunately, the values associated with present-day production facilities, particularly $\eta_{\text {conv }}$, are extremely low. A good example of this is FNAL, which creates antiprotons by means of colliding beams of relativistic protons with high-atomic number (high-Z) material targets. The protons, which are typically accelerated to energies of $120 \mathrm{GeV}\left(120 \times 10^{9}\right.$ electron volts $)$, yield a spray of particles at the collision site. Electrons, positrons and pions are the most copious products, while proton-antiproton pairs are relatively rare due to their large mass Furthermore, only a small portion of the antiprotons leave the target at the proper momentum and small enough exit angle to be magnetically focused and cooled for subsequent storage.

The performance of the overall collection process is quite low and yields about 1 antiproton per $10^{5}$ proton collisions. Multiplying acceleration energy (120 GeV/proton) by collection ratio ( $10^{5}$ proton/antiproton) yields an energy requirement of $1.2 \times 10^{16} \mathrm{eV} /$ antiproton or $1.16 \times 10^{21} \mathrm{~J} / \mathrm{g}$. Dividing this into the specific rest mass energy $c^{2}$ yields $\eta_{\text {conv }}=7.8 \times 10^{-8}$. Furthermore, FNAL facilities consume $14 \mathrm{MW}$ of power to deliver $5 \times 10^{12} 120-\mathrm{GeV}$ antiprotons every 1.5 seconds onto the production target. ${ }^{12}$ This equates to $\eta_{\text {grid }}=5 \times 10^{-3}$, and according to Eq. (3), translates to $\eta_{t o t}=3.9 \times 10^{-10}$. Substituting these values and an assumed $k_{\text {grid }}$ of \$0.10 per kW-hr into Eq. (4) yields an energy cost of $\$ 6,410$ trillion per gram of antiprotons.

Obviously, the cost of producing large quantities of antimatter (i.e., gram-scale or greater) with current facilities is excessively high! However, studies have shown that the efficiency of production based on proton/high- $Z$ material collisions can be improved substantially by optimizing proton acceleration energy and duty factor, and incorporating improved collection methods. ${ }^{13}$ It is also reasonable to assume that the energy utilization of a dedicated antiproton production facility could be made more efficient, at least to a level comparable to other new accelerator systems, ${ }^{14}$ thus yielding $\eta_{\text {grid }} \sim 10 \%$. For example, assuming a collection ratio of 1 antiproton per 20 collisions $^{15}$ and a $10 \%$ wall-plug efficiency yields an $\eta_{\text {tot }}$ of $3.9 \times 10^{-5}$. This 5 order of magnitude improvement over current capability yields a cost of $\$ 64$ billion per gram, which is roughly 10,000 times the cost of an equivalent energy load of Shuttle ET propellants. 
Such improvements would most likely require a substantial investment of 3 to 10 billion FY87 dollars for a dedicated production facility. ${ }^{13}$

It appears that as long as commercial power rates remain near current levels of $\$ 0.01$ to $\$ 0.1$ per $\mathrm{kW}-\mathrm{hr}$, the cost of producing large quantities of antimatter will be high, regardless of the extent to which efficiency can be improved. In order for large-scale production to become even remotely practical (especially at the kilogram $(\mathrm{kg})$ to metric ton $(\mathrm{mT})$ quantities envisioned for interstellar missions using "pure" antimatter rockets), power utility costs will have to drop dramatically below current levels $\left(k_{g^{\prime} u t} \ll \$ 0.1 / \mathrm{kW}\right.$-hr). This is unlikely to occur until abundant power based on a conceivably "free" resource becomes available.

The prospects for applications involving small amounts of antimatter $\left(M_{a} \sim 1 \mu \mathrm{g}\right)$, however, look much more promising. Several near-term technologies being pursued in the areas of commercial radioisotope medicines, diagnostic tomography and cancer therapy require antimatter quantities ranging from only 0.1 to 100 nanograms (ng). ${ }^{16}$ With today ${ }^{3}$ production infrastructure, the energy costs for these applications lie within the range of $\$ 640$ thousand to $\$ 640$ million.

What is more important, especially for high-energy applications such as propulsion, is the significant reduction in antimatter energy costs that could be achieved by incorporating several upgrades into FNAL and other existing facilities. As the following discussion shows, a 2 to 3-order of magnitude reduction in energy costs appears feasible and could be implemented within the next decade. Also, by incorporating existing technologies into design of a dedicated antiproton production facility, the 1- to $100-\mu \mathrm{g}$ quantities required for omniplanetary spaceflight and interstellar precursor missions based on antimatter-assisted fission/fusion would cost $\$ 64$ thousand to $\$ 6.4$ million.

\section{Antimatter Propulsion Concepts}

Approximately $2 / 3$ of the total rest mass energy of an annihilating proton-antiproton pair goes into the immediate production of charged particles. It is important to utilize the energy of these products as soon as possible after the annihilation event, before they successively decay into neutral gamma rays and unusable neutrinos. This entails either (1) heating a propellant directly through particle/fluid collisions, (2) absorbing particle energy in an intermediate material which heats a propellant, or (3) directing the highly-energetic charged pions or muons out a 
magnetic nozzle to produce thrust. We consider six different antimatter propulsion concepts. These include four "conventional" systems driven solely by annihilation energy and two "hybrid" systems powered by antimatter-assisted fission/fusion.

The simplest conventional system is the solid-core concept ${ }^{5.17}$, which uses antiprotons to heat a solid, high- $Z$, refractory metal core. Propellant is pumped into the hot core and expanded through a nozzle to generate thrust. The performance of this concept is roughly equivalent to that of the nuclear thermal rocket ( $I s p \sim 10^{3} \mathrm{sec}$ ) due to temperature limitations of the solid $(\sim 3,500 \mathrm{~K})$. However, the antimatter energy conversion and heating efficiencies are typically high due to the short mean path between collisions with core atoms $\left(\eta_{e} \sim 85 \%\right)$.

A slightly more sophisticated concept is the gaseous core ${ }^{5.6,17}$, which substitutes the lowmelting point solid with a high temperature gas, thus permitting higher operating temperatures and performance ( $I s p \sim 2 \times 10^{3} \mathrm{sec}$ ). However, the longer mean free path for thermalization and absorption results in much lower energy conversion efficiencies $\left(\eta_{e} \leq 35 \%\right)$.

The third conventional concept is the plasma-core, ${ }^{6,17}$ where the gas is allowed to ionize and operate at even higher effective temperatures. Heat loss is suppressed by magnetic confinement in the reaction chamber and nozzle. Although performance is extremely high (Isp $\left.10^{4}-10^{5} \mathrm{sec}\right)$, the long mean-free path results in very low energy utilization $\left(\eta_{e} \leq 10 \%\right)$.

The "ultimate" conventional concept is the beamed-core ${ }^{3.6,10}$, which avoids the problems of heating a secondary fluid altogether. Here, the charged products of the proton-antiproton annihilation are directly expelled out of the vehicle along an axial magnetic field. The exhaust velocities of these products are exceptionally high ( $I s p \sim 10^{7} \mathrm{sec}$ ), approaching the speed of light. Although energy utilization efficiencies are also high $\left(\eta_{e} \sim 60 \%\right)$, the flowrate and thrusts are typically very low.

The hybrid antimatter/fusion concepts differ from the conventional systems in that antiprotons are used as a driver to initiate a combined fission/fusion process in a compressed plasma or condensed material target. Practically all of the propulsive energy is derived from fusion reactions. Consequently, antimatter requirements are much lower than the pure-antimatter systems.

The first of such processes is Antimatter-Catalyzed Micro-Fission/Fusion (ACMF). ${ }^{7}$ Here, a pellet of D-T and U-238 is compressed with particle beams and irradiated with a low- 
intensity beam of antiprotons. The antiprotons are readily absorbed by the U-238 and initiate a hyper-neutronic fission process that rapidly heats and ignites the D-T core. The heated fission and fusion products expand to produce thrust, but the inherent isotropy of the flow results in a lower effective energy utilization and jet efficiency. Although additional thrust is obtained from an ablating surface that absorbs neutrons and electromagnetic radiation from the ignited pellet, the performance of this concept is lower than the plasma and beamed core rockets ( $I s p \approx 13,500$ sec). Gaidos et al. ${ }^{7}$ have shown that the interaction between the antiproton beam and target exhibits extremely high-gain yielding a ratio of fusion energy to antimatter rest mass energy $\beta$ of $1.6 \times 10^{7}$. However, energy utilization is also lower due to the isotropic expansion process $\left(\eta_{e} \sim\right.$ $15 \%$ ). Assuming a 3-order of magnitude improvement in the efficiency of producing antiprotons over current values, the net energy gain is 640 .

Another concept is Antimatter-Initiated Microfusion (AIM). ${ }^{8}$ Here, an antiproton plasma within a special Penning trap is repetitively compressed via combined electric and magnetic fields. Droplets containing D-T or D-He3 mixed with a small concentration of a metal, such as $\mathrm{Pb}-208$ or $\mathrm{U}-238$, are synchronously injected into the plasma. The main mechanism for heating the liquid droplet is antimatter-induced fission fragments which have a range of 45 microns $(\mu \mathrm{m})$ in the droplet. The power density released by the fission fragments into the D-T or D-He3 is about $5 \times 10^{13} \mathrm{~W} / \mathrm{cm}^{3}$, which is enough to completely ionize and heat the fuel atoms to fusion ignition. The heated products are directed out magnetic field lines to produce thrust. The Isp and energy efficiency for this concept are higher than ACMF (Isp $\approx 67,000 \mathrm{sec}$ and $\eta_{e} \sim 84 \%$ with D-He3, and $I s p \approx 61,000 \mathrm{sec}$ and $\eta_{e} \sim 69 \%$ with D-T). The gains $\beta$ are $10^{5}$ for D-He3 and $2.2 \times 10^{4}$ for D-T. ${ }^{8}$ Again assuming a 3-order of magnitude improvement in antiproton production efficiency, these gains are near breakeven in terms of net energy flow.

Although net energy gain is a fundamental consideration in the development of terrestrial fusion power systems, it should not be the case for in-space power sources designed for propulsion. For such applications, the mass and portability of the source are equally important to energy gain. This is where antiproton-assisted fission/fusion offers a distinct advantage over conventional fusion-based propulsion concepts.

\section{Antimatter Requirements}


We consider six reference missions which reflect ambitious robotic and manned exploration of the solar system, precursor interstellar study of phenomena outside the heliopause, and missions to our closest stellar neighbors. These reflect the data used in a recent evaluation of propulsion options for interstellar missions. ${ }^{10}$ The missions and their associated $\Delta V$ 's are shown in Table 1.

Our goal is to calculate the antimatter quantities for each of the previously described concepts as a function of mission requirements, more specifically $\Delta V$ and payload mass $M_{\text {pay }}$. We begin by taking the definition of mass ratio, $R=\left(M_{p}+M_{o}\right) / M_{o}$, and equating it to the expression for $R$ from the relativistic rocket equation. ${ }^{18}$ This yields the following relationship for propellant-to-dry-mass ratio:

$$
\frac{M_{p}}{M_{o}}=R-1
$$

where:

$$
R=\left(\frac{1+\frac{\Delta V}{c}}{1-\frac{\Delta V}{c}}\right)^{\frac{c}{2 V_{e}}}
$$

Note that $M_{p}$ includes both antimatter and propellant, and $M_{o}$ accounts for vehicle structure, systems and payload. The fraction of antimatter making up the total propellant requirement is determined by equating the actual energy introduced into the propellant with the jet energy of the exhaust, that is:

$$
\left(E_{\bar{p} p}+E_{f u s i o n}\right) \eta_{e}=\left[M_{p}-\frac{E_{\bar{p} p}+E_{f i s i o n}}{c^{2}}\right] c^{2}(\gamma-I)
$$

The left-hand side of Eq. (7) represents the combined annihilation and fusion energy applied to the exhaust, and accounts for the energy-utilization efficiency of the nuclear products. The right-hand side of Eq. (7) represents kinetic energy of the exhaust products, where the rest mass of the annihilation and fusion energy is subtracted from the total reaction mass. $E_{\bar{p} p}$ is the 
rest mass energy of the annihilation reaction and accounts for both proton and antiproton reactants, $E_{\bar{p} p}=2 m_{t} c^{2}$. The fusion energy is expressed in terms of annihilation energy with $E_{\text {fisision }}=\beta E_{\bar{p} p}$.

Substituting the definitions of $E_{\bar{p} p}$ and $E_{\text {fusion }}$ into Eq. (7) and rearranging terms yields an expression for the antimatter-to-propellant-mass ratio. This in turn can be multiplied by Eq. (5) to yield an expression for antimatter-to-inert-mass ratio as a function of mission requirements, propulsion performance, fusion gain and energy efficiency:

$$
\frac{M_{u}}{M_{o}}=\frac{1-}{2(1+\beta)}\left[\frac{\gamma-1}{\eta_{\underline{\underline{e}}}+\gamma-1}\right](R-1) \text {. }
$$

Inert mass can be expressed in terms of payload mass by using the definition for structure to propellant ratio, $\lambda=M_{\text {struct }} / M_{\text {prop }}$, where $M_{\text {struct }}=M_{o}-M_{\text {pay }}$ :

$$
M_{o}=\left(\frac{1}{1+\lambda-\lambda R}\right) M_{\text {pay }}
$$

Substituting Eq. (9) into Eq. (8) yields the following expression for $M_{a}$ in terms of $M_{p a y}$ and propulsion/performance parameters:

$$
M_{a}=\frac{1}{2(1+\beta)}\left(\frac{\gamma-1}{\gamma+\left(\eta_{e}-1\right)}\right)\left(\frac{R-1}{1+\lambda-R \lambda}\right) M_{p a y}
$$

We use Eq. (10) to illustrate in Fig. 1 the dependence of antimatter mass on payload and mission velocity ${ }^{10}$ for the ACMF, AIM, plasma core and beamed core concepts. Plots for the solid- and gas-core concepts are not shown, because the Isp's are lower than either of the hybrid concepts, and the antimatter requirements are only marginally less than that of the plasma core. For these calculations, values of $\lambda$ were synthesized from estimates by various sources. ${ }^{4-10,16} \mathrm{~A}$ $\lambda$-value of 0.3 was assumed for both the solid- and gas-core concepts to account for tankage, structure and reaction containment (i.e., core, confinement chamber). The $\lambda$-values for the plasma core, beamed core and AIM were lower $(\lambda=0.2)$ because of the improved reaction 
confinement performance expected with these more advanced concepts. With the ACMF concept, a much higher $\lambda$ of 0.7 was assumed to account for the large mass of the ion driver system.

In the lower range of mission velocities ( $10 \mathrm{~km} / \mathrm{sec} \leq \Delta V \leq 10^{3} \mathrm{~km} / \mathrm{sec}$ ), the ACMF and AIM concepts are clearly superior in terms of minimizing antimatter requirements. For planetary, early interstellar precursor and simple omniplanetary applications, ACMF exhibits the best performance. The reference case of a 1-year human round-trip mission to Jupiter with a 10 to 100 metric ton $(\mathrm{mT})$ payload requires an antimatter quantity of 1 to 10 micrograms $(\mu \mathrm{g})$. It appears as though this requirement could drop into the 1 to $10 \mathrm{ng}$ range for payloads consistent with unmanned, planetary missions. However, ACMF was originally conceived for crewed omniplanetary flight and is probably not scaleable to smaller sizes due to the large mass of its ion driver system. Therefore, $\mathrm{ACMF}$ is restricted to missions which would require 1 to $10 \mu \mathrm{g}$ and $\Delta V$ 's less than or equal to $100 \mathrm{~km} / \mathrm{sec}$.

The AIM concept, which does not need a driver and benefits from a higher Isp, can accomplish more ambitious missions, such as interstellar precursor trips to the Oort cloud. However, the antimatter requirement is roughly 1 to 2 orders of magnitude greater than ACMF. For that reason, this concept is better suited for unmanned missions with smaller payloads. The design point in Fig. 1 represents a 50-year trip to the Oort cloud with a $100 \mathrm{~kg}$ payload. Even with the higher rate of antiproton usage, the total requirement is still relatively low, within the 10 to $100 \mu \mathrm{g}$ range. The structural ratio and $I s p$ limit the maximum $\Delta V$ to $10^{3} \mathrm{~km} / \mathrm{sec}$.

The only antimatter concept that can achieve velocities above $2 \times 10^{3} \mathrm{~km} / \mathrm{sec}$ and accomplish missions well beyond solar influence is the beamed core. Although a structural ratio consistent with the AIM and plasma core concepts is assumed, the much higher exhaust velocity of the annihilation products permits vehicle accelerations to velocities approaching $0.4 \mathrm{c}$, which would enable "fast" missions to Alpha Centauri in 10 years. ${ }^{10}$ At first this appears quite attractive until one notes that the antimatter requirement is many orders of magnitude greater than either the ACMF or AIM reference case. For a payload of 1 metric ton (mT), the antimatter requirement is about $40 \mathrm{mT}$, depending on the mission. The beamed core requires tremendous amounts of antimatter, but it is the only concept that can travel to the nearest stars (i.e., 4 to 40 light years) within a "reasonable" time (i.e., 10 to 100 years). 
Although the inordinately high antimatter requirements of the conventional systems are impractical to consider in the near-term, the more modest quantities associated with ACMF and AIM may be quite attainable. The catalyzed systems could not be used for trips to the stars, due to their limited $\Delta V$ 's of only $10^{2}$ to $10^{3} \mathrm{~km} / \mathrm{sec}$. However, ACMF and AIM appear to have sufficient performance to propel interstellar precursor probes and support human exploration of the entire solar system.

\section{Antimatter Production Capability}

Almost all of the controlled antimatter in the world is produced at either CERN or FNAL. Figure 2 shows a schematic of the accelerator system currently used at FNAL for producing antiprotons. ${ }^{19}$ Protons from an $\mathrm{H}^{-}$source are sequentially accelerated to $120 \mathrm{GeV}$ in the Main Injector. These protons then collide with a target, which produces antiprotons and a plethora of other fundamental particles. The antiprotons are sign-selected with magnets and accumulated in the $8-\mathrm{GeV}$ Antiproton Source. Here they are stochastically cooled and temporarily held within the storage ring. For physics experiments these antiprotons are re-injected as intense beams back into the Main Ring in order to carry out collisions with protons at very high energies. For future space propulsion applications, the $8-\mathrm{GeV}$ antiprotons could be loaded into a new ring (not shown) and decelerated to an energy low enough to allow collection in traps.

This unique capability was added from the late-70's to the late-90's to increase the energies of particle collision experiments. During a year-long period between 1997 and 1998, FNAL produced $1 \mathrm{ng}$ of antiprotons. This was done in the midst of a very large experimental program that did not have sufficient funds to run 24 hours per day, 365 days per year. The instantaneous accumulation rates were around $10^{11}$ antiprotons/hour, so a full year of operation would have produced $8.8 \times 10^{14}$ antiprotons. This equates to an annual yield of approximately $1.5 \mathrm{ng}$, which is 3 to 4 orders of magnitude less than the quantities required for missions using $\mathrm{ACMF}$ and AIM.

It is important to remember that neither of the facilities at FNAL or CERN was designed for the sole purpose of producing antiprotons. This capability, which was added after the facilities had been operating for some time, was only intended to generate enough antiprotons for collision experiments. The collection ratio, which can be viewed as the effective antiproton yield (i.e., antiprotons collected) per proton on target, was not the main concern. Although the current ratio of $10^{-5}$ is very low, there are ways in which it can be increased. 
We consider the case of FNAL, which is the largest, most convenient source of antiprotons in the U.S. From 1998 to mid-1999, FNAL's accelerator was down for commissioning of a new Main Injector. We expect that when the new injector comes on-line in 1999, production yields will increase by another order of magnitude. This will eventually boost the production rate to about $10^{12}$ antiprotons/hour. We therefore expect that by the early part of next decade, the total annual production capacity should approach $15 \mathrm{ng}$. At the same time, FNAL could start incorporating even better collection devices and techniques. Development of more efficient collection equipment, such as improved focusing horns and multiple large-aperture receivers, has been considered, ${ }^{13}$ and could culminate in substantial production gains. It is quite reasonable to expect perhaps an additional 50-fold increase in efficiency with these upgrades, thus yielding a 500-fold improvement over current capability.

The impact of incorporating such improvements is shown in Fig. 3. The final result is a nearly 3-order of magnitude increase of production capacity into the microgram-range. This is significant because at this level one can seriously begin to consider use of antimatter-catalyzed fusion propulsion devices for space applications.

These production enhancements are obviously aimed at expanding support of scientific research at FNAL. However, customers who are planning to use the facility for replenishment of portable antiproton devices, such as NASA and commercial users, would require an additional feature beyond those planned to support scientific activities.

In the current production process, high-energy antiprotons from the original proton collision site can be stored temporarily in the Main Injector at a relatively low kinetic energy of $433 \mathrm{MeV}$. They are subsequently extracted and accelerated to much higher energies for collision experiments. To transfer these antiprotons into a small-volume, portable device, such as a Penning trap, an additional deceleration process, which would reduce antiproton energies from $433 \mathrm{MeV}$ to no more than $20 \mathrm{keV}$, is required between the Main Injector and storage device. ${ }^{20}$

The development of antiproton Penning traps has progressed extremely well over the last 10 years. The PS200 experiment ${ }^{21}$ trapped over $10^{6}$ antiprotons for periods of hours. This is seen as a means of soon being able to confine up to $10^{12}$ antiprotons with transfer to a remote site for periods of several months. ${ }^{22}$ Work is currently underway for development of a magnetic degrading spectrometer which will simply and inexpensively decelerate antiprotons into such portable traps. This approach is adequate for some important commercial applications and 
demonstrating fundamental propulsion concepts, such as generation of subcritical microfission reactions and plasma formation as a precursor to fusion reactions. However, it will not be capable of providing the much larger quantities needed for direct propulsion applications. In this case, a more efficient decelerator section will be required to achieve production rates equivalent to $\sim 1 \mu$ g per year. Antiproton decelerators which accomplish this do exist (e.g., at CERN), and in the case of FNAL would cost about $\$ 10$ million to construct.

If the anticipated demand from the scientific community, NASA and the commercial sector continues to grow, then investment in a completely new production-oriented facility would probably be warranted. ${ }^{23}$ In the 1980 's, the RAND Corporation studied development of such a capability and concluded that a capacity of several milligrams per year to possibly 1 gram per year could be achieved with a new machine costing 3 to 10 billion FY87 dollars. ${ }^{13}$ Two types of facilities were considered (i.e., fixed target and colliding beams as illustrated in Fig. 4) both capable of producing annual antiproton yields in the milligram to gram range. A comprehensive research and development program required to implement such systems was considered. Among the topics addressed were accelerator-types (including intense, rapid cycling synchotrons and high repetition-rate linacs), targeting, cooling of particle beams, plasma collection lenses, and large aperture collector rings.

It is important to note that a multi-billion dollar investment in such a capability is consistent with that of some previous major national science projects, and the design of such a facility falls well within the realm of known technology. In fact, the basic production process would be very similar to the current method of creating antiprotons from collisions of protons with high- $Z$ targets. However, improvements, such as higher- $Z$ accelerated particles and more efficient collection/focusing devices, would enhance efficiency considerably.

For capacities above 1 gram, which would support a highly-evolved transportation infrastructure within the solar system and trips into interstellar space, a completely new production technology is necessary. Several methods look promising, but all are at the very early stages of technological maturity.

Other issues are how to store groups of antiprotons of this scale and containment of the stored energy on this scale. Again, the energy stored within 1 gram of antimatter is roughly equivalent to that delivered by 23 Shuttle ET's. A systematic approach to safe storage of such quantities is required, as has been done with other highly energetic and reactive materials. Studies 
of high-density storage of antimatter are underway and are an important step along this critical pathway.

\section{Antimatter Production Costs}

The costs of producing batches of antimatter on demand are not well characterized, since the facilities do not yet provide this function as an actual service. FNAL is beginning to recognize the existence of an incipient demand outside the high-energy physics community. Although less experienced than FNAL, Brookhaven National Laboratory has recently expressed interest in "going into the antimatter business"; however, Brookhaven's facilities are much less developed than those at FNAL.

From our previoùs analysis, the current cost of producing $1 \mu \mathrm{g}$ of antimatter is $\$ 6.4$ billion. Assuming present production levels, the antimatter needed to support highly ambitious ACMF or AIM missions $(\sim 100 \mu \mathrm{g})$ would cost $\$ 640$ billion, much too high for practical considerations. In addition, the extremely low production rate would require an unreasonably long fill time on the order of 100's of years. The situation looks discouraging until we account for the anticipated improvements to the current production capacity. In this case the costs would go down by at least 2 orders of magnitude to $\$ 64$ million per $\mu \mathrm{g}$ or $\$ 6.4$ billion for a $100 \mu \mathrm{g}$ mission. This is too expensive to support even occasional missions, and is certainly prohibitive for anything above the $10 \mu \mathrm{g}$ level. However, this cost certainly permits ground-based testing and demonstration of antimatter-assisted fusion/fission propulsion technology, which would require quantities of only $1 \mu \mathrm{g}$ or less.

For actual missions, especially regular excursions to the outer solar system, there will be a need for investment in a new, dedicated facility. For antimatter requirements in the $\sim 1$ milligram range and above, costs would have to be based on the capabilities of a new facility. In the previous section, the initial cost for such a capability was estimated to be $\$ 3$ to $\$ 10$ billion. ${ }^{13}$ Production efficiencies would be much greater. Assuming an $\eta_{t o t}$ of $3.9 \times 10^{-5}$ and the power grid costs from before, the costs could come down by nearly 3 more orders of magnitude to $\sim \$ 64$ thousand per $\mu \mathrm{g}$. In this case the antimatter cost for a $100 \mu \mathrm{g}$ mission would be $\$ 6.4$ million. At such values, antimatter becomes affordable enough to support a space transportation infrastructure based on some form of antimatter-assisted fission/fusion. 


\section{Conclusions}

We have completed a study that: (1) evaluated the antimatter requirements for various propulsion concepts over a range of missions and velocity requirements, (2) compared these requirements against the capabilities of the existing antimatter production infrastructure, (3) compared these again assuming the improved capability expected over the next several years, and (4) estimated antimatter costs in \$/microgram for both the current and improved infrastructure.

Results show that the antimatter costs associated with conventional antimatter rockets, that is systems which rely on antimatter as the sole source of propulsive energy, are too high to be seriously considered for anything other than missions to nearby stars. Even missions within the solar system and into near-interstellar space would require production rates 6 to 9 orders of magnitude greater than the existing infrastructure.

Antimatter-assisted fission/fusion, however, holds considerable promise for near-term applications. Although this form of propulsion could not be used for trips to the stars, it does provide excellent performance for missions within the solar system and near-interstellar space. The requirements for antimatter are on the scale of 1 to $100 \mu \mathrm{g}$ per mission, which with the current infrastructure equates to an antiproton cost of $\$ 6.4$ billion to $\$ 640$ billion. However, with several upgrades that could be incorporated in the near-term, the cost could drop by at least 2 orders of magnitude to $\$ 64$ million per $\mu \mathrm{g}$. This would enable development and demonstration of these technologies, which could justify investment in a dedicated facility based on existing production technology. Such a facility could support mission requirements at a cost of $\$ 64$ thousand to $\$ 6.4$ million per mission. These costs are certainly within the range of economic feasibility, and suggest that antimatter-assisted fission/fusion may be a viable "first step" in applying antimatter for space propulsion. 


\section{References}

1. Sanger, E., "The Theory of Photon Rockets," Ing. Arch., Vol 21, 1953.

2. Morgan, D.L.. "Concepts for the Design of an Antimatter Annihilation Rocket," J. British Interplanetary Society, Vol 35.9, 1982.

3. Forward, R.. "Antiproton Annihilation Propulsion," J. Propulsion and Power, Vol 1.5, 1985.

4. Cassenti, B.N., "Design Considerations for Relativistic Antimatter Rockets," J. British Interplanetary Society, Vol 35, 1982.

5. Howe, S.D. and Metzger, J.D., "Antiproton-Based Propulsion Concepts and the Potential Impact on a Manned Mars Mission," J. Propulsion and Power, Vol. 5.3, 1989.

6. Cassenti, B.N., "High Specific Impulse Antimatter Rockets," AIAA 91-2548, June 1991.

7. Gaidos, G., Lewis, R.A., Smith, G.A., Dundore, B. and Chakrabarti, S. "AntiprotonCatalyzed Microfission/Fusion Propulsion Systems for Exploration of the Outer Solar System and Beyond," AIAA 98-3589, July 1998; Gaidos, G., Laiho, J., Lewis, R.A., Smith, G.A., Dundore, B. and Chakrabarti, S.. Space Technology and Applications International Forum - 1998, ed. M.S. El-Genk, AIP Conf. Proc. 420, p 1365.

8. Gaidos, G., Lewis, R.A., Meyer, K., Schmidt, T. and Smith, G.A., "AIMStar: Antimatter Initiated Microfusion for Precursor Interstellar Missions," Space Technology and Applications International Forum - 1999, ed. M.S. El-Genk, AIP Conf. Proc. 458, p 954; Acta Astronautica, Vol 44, 1999, p 183.

9. Cassenti, B., Kammash, T. and Galbraith, D.L., "Antiproton-Catalyzed Fusion Propulsion for Interplanetary Missions," AIAA 96-3068, July 1996.

10. Frisbee, R.H. and Leifer, S.D., "Evaluation of Propulsion Options for Interstellar Missions," AIAA 98-3403, July 1998.

11. Rider, T.H., "Fundamental Constraints on Large-Scale Antimatter Rocket Propulsion," J. Propulsion and Power, Vol 13.3, May-June 1997.

12. Antiproton production facilities presently consume $14 \mathrm{MW}$ of power at FNAL, delivering 5 x $10^{12}$ protons every 1.5 seconds at $120 \mathrm{GeV}$ energy onto the production target. (J. Marriner, private communication.)

13. "Proceedings of the RAND Workshop on Antiproton Science and Technology," World Scientific, Singapore, New Jersey and Hong Kong, June, 1988; Augenstein, B.W., Annotated Executive Summary," RAND Note N-2763-AF, October 1988. 
14. Accelerator Production of Tritium (APT), under development at LANL. would provide, if completed. $100 \mathrm{~mA}$ of protons at $1.6 \mathrm{GeV}$ energy on target with a wall-plug efficiency of $42 \%$. The value of $42 \%$ may not be verified, since DoE has recently decided to use commercial power reactors to make tritium. (S. Howe/G. Lawrence, private communication.)

15. Forward, R.L., "Advanced Space Propulsion Study - Antiproton and Beamed Power Propulsion," Air Force Astronautics Laboratory, Report AD-A189-218, Oct. 1987; Forward, R.L., "Prospects for Antiproton Production and Propulsion," Proceedings of the Cooling, Condensation, and Storage of Hydrogen Cluster Ions Workshop, SRI International, Menlo Park, CA, January 1987.

16. Lewis, R.A., Smith, G.A. and Howe, S.D., "Antiproton Portable Traps and Medical Applications," Hyperfine Interactions, Vol 109, 1997.

17. Borowski, S.K., "Comparison of Fusion/Antiproton Propulsion Systems for Interplanetary Travel," Fusion Energy in Space Propulsion: AIAA Progress in Astronautics and Aeronautics, Vol 167, Kammash, T. ed., 1995.

18. Mallove, E. and Matloff, G., "The Starflight Handbook - A Pioneer's Guide to Interstellar Travel," John Wiley \& Sons, Inc., 1989.

19. Jackson, G.P., "An Intense Source of Antiprotons for Antimatter Research at FNAL," Hyperfine Interactions, Vol. 109, 1997.

20. Holzscheiter, M.H., Lewis, R.A., Mitchell, E., Rochet, J. and Smith, G.A., Production and Trapping of Antimatter for Space Propulsion Applications," Space Technology and Applications International Forum - 1997, ed. M.S. El-Genk, AIP Conf. Proc. 387, p 1493.

21. Holzscheiter, M.H. et al, "Are Antiprotons Forever?" Physics Letters A, Vol 214, 1996.

22. Smith, G.A. and Meyer, K.J., "Preliminary Design for the High Performance Antimatter Trap (HiPAT)," NASA Marshall Space Flight Center, 1998; Smith, G.A. and Kramer, L.J., "Enabling Exploration of Deep Space: High Density Storage of Antimatter," NASA Marshall Space Flight Center, 1999.

23. Cassenti, B.N., "Concepts for the Efficient Production and Storage of Antimatter," AIAA 93-2031, June 1993. 
Table 1: Reference Missions

\begin{tabular}{|c|c|c|}
\hline Mission & Description & Typical $\Delta V(\mathrm{~km} / \mathrm{s})$ \\
\hline Planetary & $\begin{array}{l}\text { Deep space robotic missions } \\
\text { throughout solar system }\end{array}$ & 10 \\
\hline Omniplanetary & $\begin{array}{l}\text { Ambitious human exploration } \\
\text { throughout solar system }\end{array}$ & $30-200$ \\
\hline $100-1000 \mathrm{AU}$ & $\begin{array}{l}\text { Interstellar precursor missions to } \\
\text { - Heliopause (100 AU) } \\
\text { - Gravity Lens focus (550 AU) }\end{array}$ & 100 \\
\hline $10,000 \mathrm{AU}$ & $\begin{array}{l}\text { Interstellar precursor mission to } \\
\text { Oort Cloud }(10,000 \mathrm{AU})\end{array}$ & 1,000 \\
\hline Slow Interstellar & 4.5 light-years in 40 years & $\begin{array}{l}30,000 \\
(=0.1 \mathrm{c})\end{array}$ \\
\hline Fast Interstellar & $\begin{array}{l}4.5 \text { light-years in } 10 \text { years or } 40 \\
\text { light-years in } 100 \text { years }\end{array}$ & $\begin{array}{l}120,000 \\
(=0.4 c)\end{array}$ \\
\hline
\end{tabular}




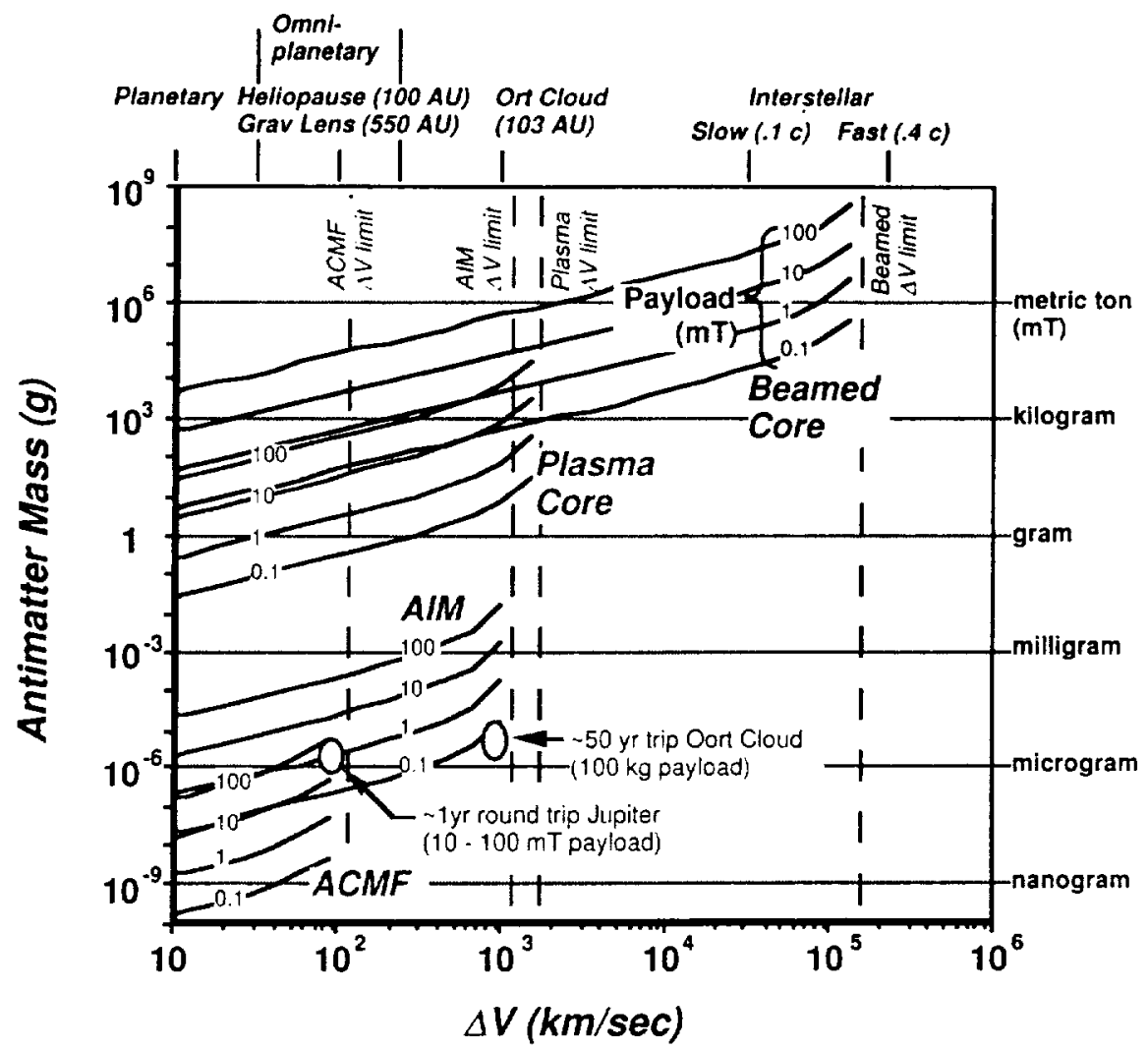

Figure 1: Antimatter requirements for different propulsion concepts 


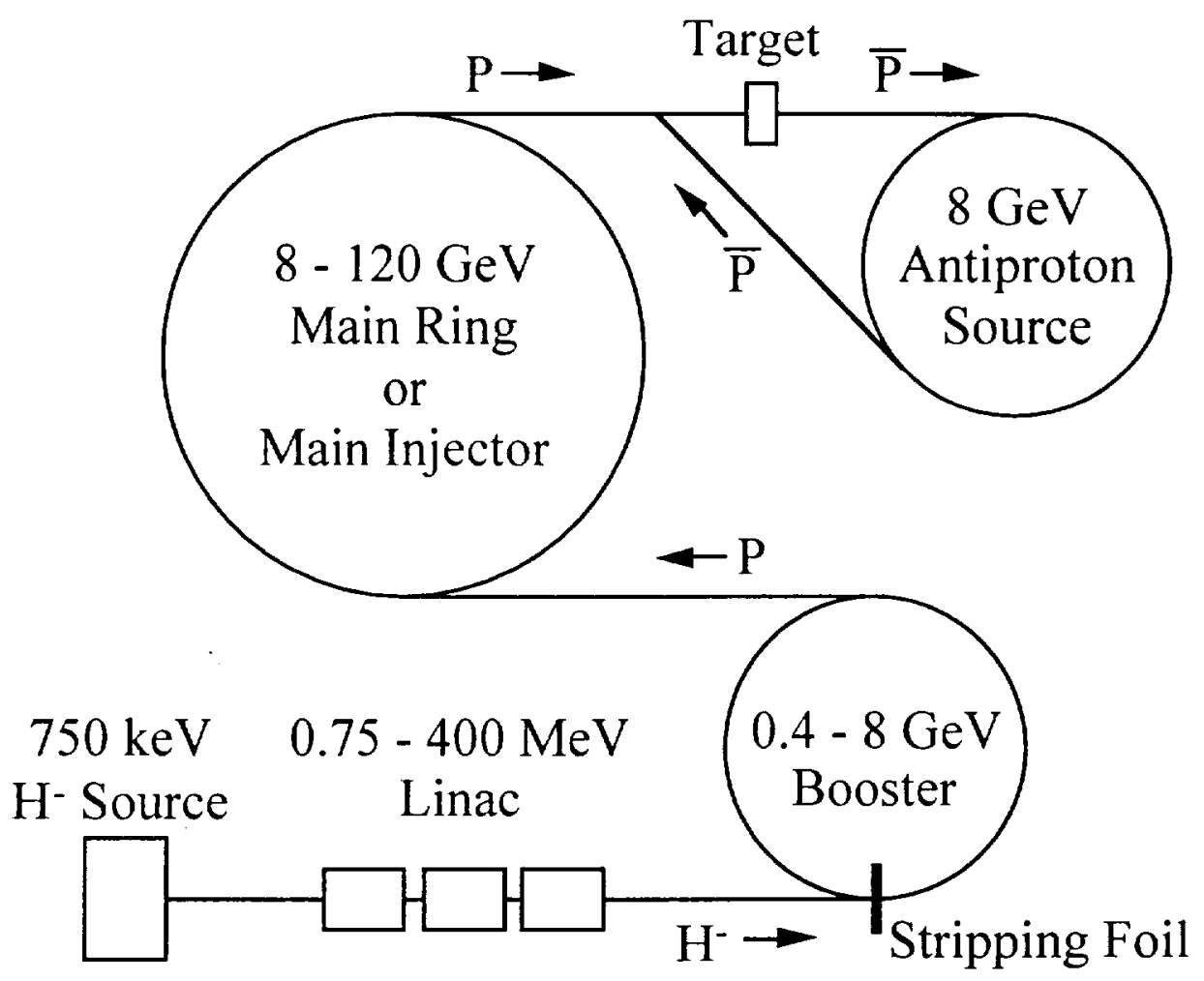

Figure 2: Antiproton production facilities at FNAL [Ref. 19] 


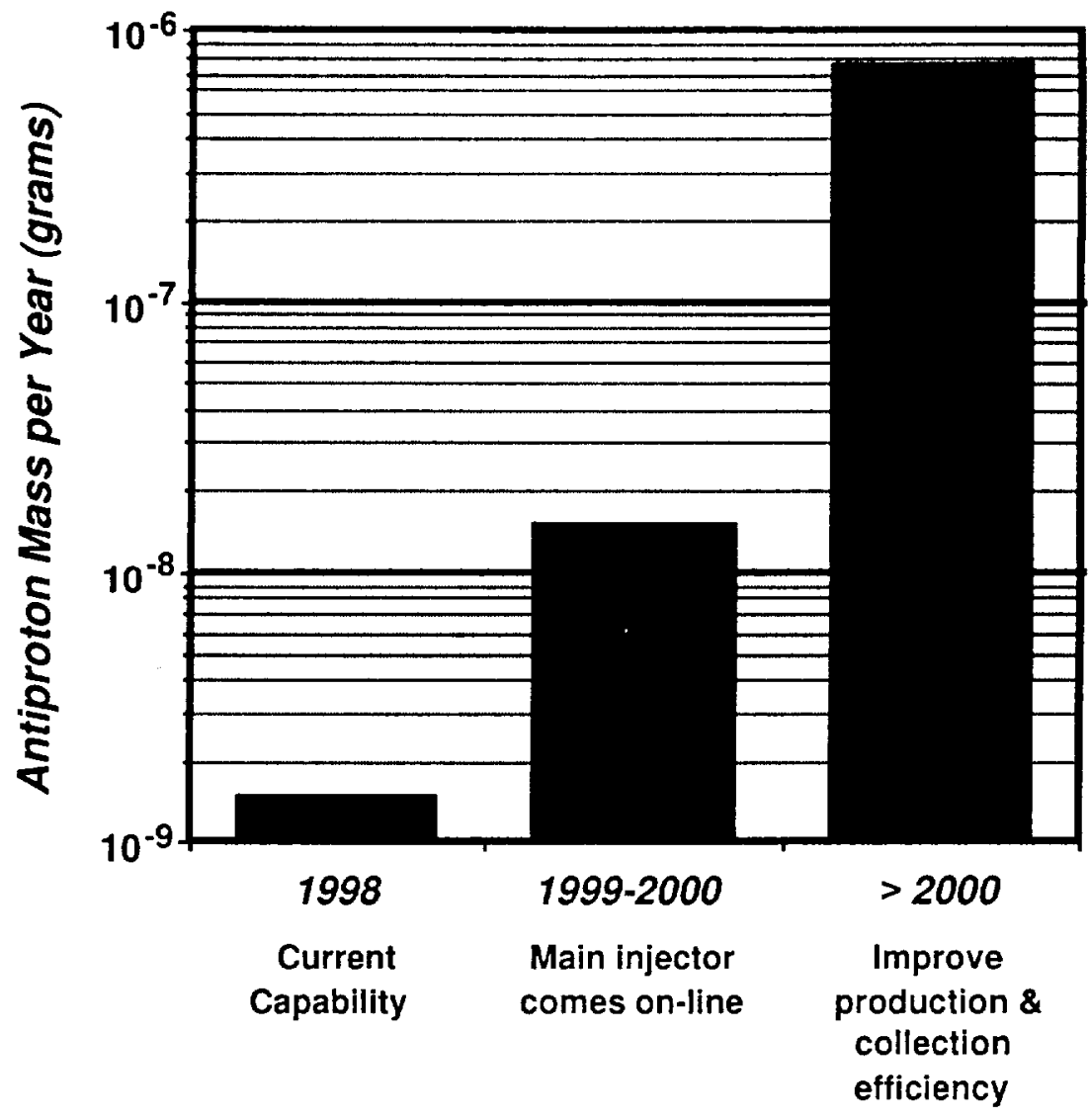

Figure 3: Impact of near-term improvements at FNAL 
Fixed target, enveloping plasma collector

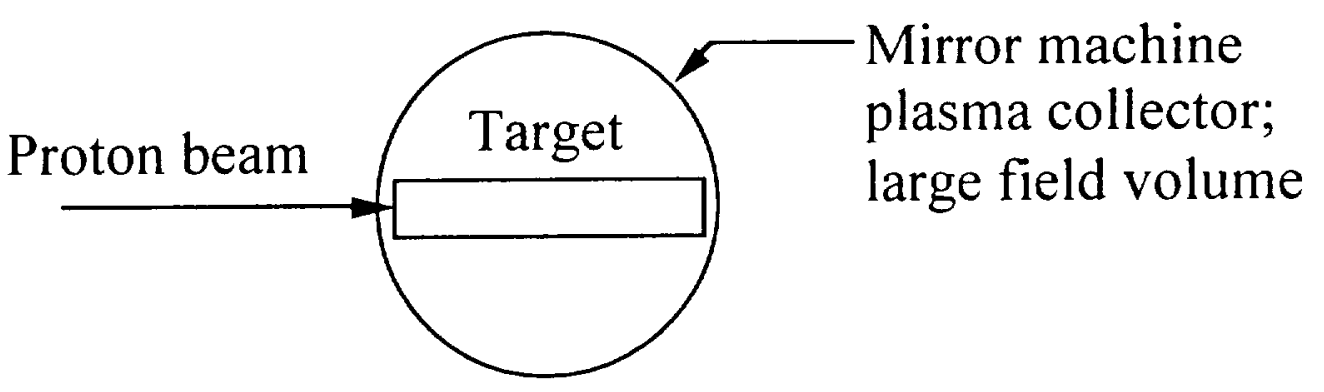

\section{Colliding beams, enveloping plasma collector}

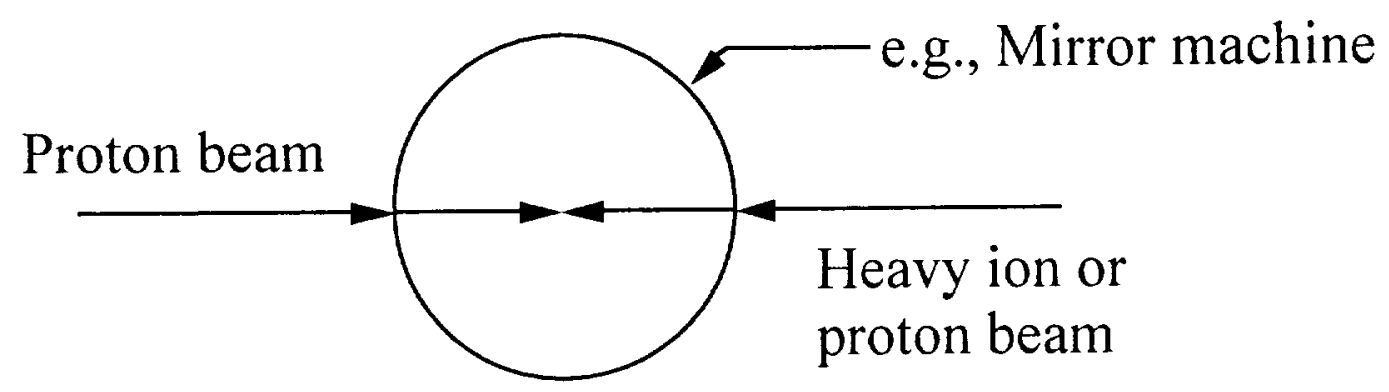

Figure 4: Concepts for milligrams per year production facility [Ref. 13] 